\title{
Erratum to: Real-Imaginary Conjugacy Classes and Real-Imaginary Irreducible Characters in Finite Groups*
}

\author{
A. Beltrán ${ }^{1 * *}$ and S. M. Robati ${ }^{2 * * *}$ \\ ${ }^{1}$ Departamento de Matemáticas, Universidad Jaume I, Castellón, 12071 Spain \\ ${ }^{2}$ Department of Pure Mathematics, Faculty of Science, Imam Khomeini International University, \\ Qazvin, 34148-96818 Iran
}

Received October 8, 2020; in final form, October 8, 2020; accepted September 15, 2021

DOI: $10.1134 / \mathrm{S} 0001434621090352$

The original article can be found online at https://doi.org/10.1134/S0001434618010261

Let $G$ be a finite group. A character $\chi$ of $G$ is said to be real-imaginary if its values are real or purely imaginary. A conjugacy class $C$ of $a$ in $G$ is real-imaginary if and only if $\chi(a)$ is real or purely imaginary for all irreducible characters $\chi$ of $G$. And a finite group $G$ is called real-imaginary if all of its irreducible characters are real-imaginary.

In [1], the author described real-imaginary conjugacy classes and real-imaginary characters. In Theorem 1 of [1], he showed that $C$ is a real-imaginary conjugacy class of $G$ if and only if $C^{2}=\left(C^{-1}\right)^{2}$. In the next example, we show that this statement is not true.

Example. Let $G$ be a non-Abelian group of order 21, i.e.

$$
G=\left\langle a, b \mid a^{7}=b^{3}=1, b^{-1} a b=a^{2}\right\rangle .
$$

We can check easily from the character table of $G$ that the conjugacy class $C=\left\{a, a^{2}, a^{4}\right\}$ satisfies $C^{2}=\left(C^{-1}\right)^{2}$, but it is not real-imaginary.

The mistake in the proof of Theorem 1 of [1] comes from the last paragraph of p. 252, in which it was asserted that if $C$ is the conjugacy class of $a$ in $G$ such that $C^{2}=\left(C^{-1}\right)^{2}$, then $\chi(a) \chi(a)$ is real for each irreducible character $\chi$ of $G$. As a counterexample to this claim, take the above example and the character $\chi \in \operatorname{Irr}(G)$ that takes the following values: $\chi(1)=3, \chi(a)=\alpha+\alpha^{2}+\alpha^{4}\left(\alpha^{7}=1\right)$; $\chi\left(a^{6}\right)=\alpha^{3}+\alpha^{5}+\alpha^{6}$; and $\chi(g)=0$ for every element $g \in G$ of order 3 . It follows from Problem 3.12 of [2] that

$$
\chi(a) \chi(a)=\frac{\chi(1)}{|G|} \sum_{x \in G} \chi\left(a a^{x}\right)=\frac{\chi(1)}{|G|}\left(7 \chi\left(a^{3}\right)+7 \chi\left(a^{5}\right)+7 \chi\left(a^{2}\right)\right),
$$

which is not real.

The following theorem is the revised version of Theorem 1 of [1].

Theorem 1. Let $G$ be a finite group, let $C$ be a conjugacy class of $G$, and let $\mathfrak{C}$ denote the class sum of $C$ in the complex group algebra $\mathbb{C}[G]$. Then $C$ is a real-imaginary conjugacy class if and only if $\mathfrak{C}^{2}=\left(\mathfrak{C}^{-1}\right)^{2}$.

\footnotetext{
${ }^{*}$ The article was submitted by the authors for the English version of the journal.

${ }^{* *}$ E-mail: abeltran@uji.es

${ }^{* * *}$ E-mail: mahmoodrobati@sci.ikiu.ac.ir, sajjad.robati@gmail.com
} 
Proof. Assume that $C=a^{G}$ is a real-imaginary conjugacy class, i.e., $\chi(a)$ is real or purely imaginary for all $\chi \in \operatorname{Irr}(\mathrm{G})$. By Theorem 2.4 of [2], we see that $\mathfrak{C}^{2}=\sum_{j} a_{j} \mathfrak{C}_{j}$ and $\left(\mathfrak{C}^{-1}\right)^{2}=\sum_{j} b_{j} \mathfrak{C}_{\mathrm{j}}$, where $\mathfrak{C}$, $\mathfrak{C}^{-1}$, and $\mathfrak{C}_{j}$ are the class sums of the conjugacy classes $C, C^{-1}=\left(a^{-1}\right)^{G}$, and $C_{j}$ in $G$. By Problem 3.9 from [2], we can write

$$
a_{j}=\frac{|C|^{2}}{|G|} \sum_{\chi \in \operatorname{Irr}(\mathrm{G})} \frac{\chi(a)^{2} \overline{\chi\left(c_{j}\right)}}{\chi(1)}=\frac{\left|C^{-1}\right|^{2}}{|G|} \sum_{\chi \in \operatorname{Irr}(\mathrm{G})} \frac{\chi\left(a^{-1}\right)^{2} \overline{\chi\left(c_{j}\right)}}{\chi(1)}=b_{j}
$$

where $c_{j} \in C_{j}$ for every $j$. Thus, the equality $\mathfrak{C}^{2}=\left(\mathfrak{C}^{-1}\right)^{2}$ is proved.

For the converse assertion, suppose that $\mathfrak{C}^{2}=\left(\mathfrak{C}^{-1}\right)^{2}$; let $\chi \in \operatorname{Irr}(G)$, and let $\mathfrak{X}$ be a complex irreducible representation associated to $\chi$. Following the notation of [2], we know that $\mathfrak{X}$ can be linearly extended to $\mathbb{C}[G]$ and that $\mathfrak{X}(\mathfrak{C})=w_{\chi}(\mathfrak{C}) I$, where

$$
w_{\chi}(\mathfrak{C})=\frac{|C| \chi(a)}{\chi(1)}
$$

and $I$ is the identity matrix. Since $\mathfrak{C}^{2}=\left(\mathfrak{C}^{-1}\right)^{2}$, we have $w_{\chi}(\mathfrak{C}) w_{\chi}(\mathfrak{C})=w_{\chi}\left(\mathfrak{C}^{-1}\right) w_{\chi}\left(\mathfrak{C}^{-1}\right)$, and hence

$$
\frac{|C| \chi(a)}{\chi(1)} \frac{|C| \chi(a)}{\chi(1)}=\frac{\left|C^{-1}\right| \chi\left(a^{-1}\right)}{\chi(1)} \frac{\left|C^{-1}\right| \chi\left(a^{-1}\right)}{\chi(1)} \text {. }
$$

This gives

$$
\chi(a)^{2}=\chi\left(a^{-1}\right)^{2}=\overline{\chi(a)}^{2}
$$

for every $\chi \in \operatorname{Irr}(G)$, and this is certainly equivalent to the fact that $\chi(a)$ is real or purely imaginary for every $\chi \in \operatorname{Irr}(G)$. Therefore, $C$ is a real-imaginary class, as wanted.

Remark. The equality $(\mathfrak{C})^{2}=\left(\mathfrak{C}^{-1}\right)^{2}$ trivially implies the set equality $C^{2}=C^{-2}$, but the converse is not necessarily true. Consequently, the condition $C^{2}=C^{-2}$ is not enough for a conjugacy class $C$ to be real-imaginary. In the example, the conjugacy class $C$ satisfies $C^{2}=C^{-2}$, but $(\mathfrak{C})^{2} \neq\left(\mathfrak{C}^{-1}\right)^{2}$. Indeed, we have

$$
\left(\mathfrak{C}^{\mathfrak{c}}\right)^{2}=\mathfrak{C}+2 \mathfrak{C}^{-1}, \quad\left(\mathfrak{C}^{-1}\right)^{2}=2 \mathfrak{C}+\mathfrak{C}^{-1}
$$

and $C$ is not real-imaginary.

\section{FUNDING}

The first author was partially supported by the Spanish Government, Proyecto PGC2018-096872B-I00, by Generalitat Valenciana, Proyecto AICO-2020-298, and also by Proyecto UJI-B2019-03.

\section{REFERENCES}

1. S. M. Robati, "Real-imaginary conjugacy classes and real-imaginary irreducible characters in finite groups," Math. Notes 103 (2), 251-258 (2018).

2. I. M. Isaacs, Character Theory of Finite Groups, Academic Press, New York-San Francisco-London (1976). 